\title{
Perioperative Care of a Child With Cri Du Chat Syndrome
}

\author{
Catherine Davis $^{\mathrm{a}, \mathrm{d}}$, Jonathan Grischkan ${ }^{\mathrm{b}}$, Joseph D. Tobias ${ }^{\mathrm{c}}$
}

\begin{abstract}
Cri du chat syndrome (CdCS) is a chromosomal disorder resulting from a deletion in the short arm of chromosome 5. Anatomical abnormalities of the larynx result in a distinctive high-pitched, cat-like cry for which the disorder is named. Typical findings of the syndrome involve the upper airway, cardiovascular, and central nervous system (CNS). Of particular concern during anesthetic care is the potential for airway abnormalities leading to difficulties with endotracheal intubation as well as the presence of congenital heart disease (CHD). We present a 15-month-old child with CdCS who required anesthetic care during direct laryngoscopy and supraglottoplasty. The perioperative concerns of such patients are discussed, and previous reports of anesthetic care reviewed.
\end{abstract}

Keywords: Cri du chat syndrome; Cat cry syndrome; 5 p minus syndrome; Anesthesia; Perioperative care

\section{Introduction}

Cri du chat syndrome (CdCS), also known as $5 p$ minus syndrome or cat cry syndrome, is a chromosomal disorder that was first described by Lejeune et al in 1963 [1]. A total or partial deletion of the short arm of chromosome 5 (5p-) results in an anatomically abnormal larynx causing the distinctive highpitched, cat-like cry for which the disorder is named [2]. Additional phenotypic characteristics include microcephaly, facial dysmorphism (epicanthus, wide nasal bridge, short philtrum, flattened maxilla, micrognathia, hypertelorism), hypotonia, intellectual disability with developmental delay, and congenital

Manuscript submitted May 12, 2020, accepted May 19, 2020

Published online August 6, 2020

${ }^{a}$ Heritage College of Osteopathic Medicine, Dublin, OH, USA

${ }^{b}$ Department of Otolaryngology and Head and Neck Surgery, Nationwide Children's Hospital, The Ohio State University College of Medicine, Columbus, $\mathrm{OH}, \mathrm{USA}$

'Department of Anesthesiology and Pain Medicine, Nationwide Children's Hospital, The Ohio State University College of Medicine, Columbus, OH, USA

${ }^{\mathrm{d} C o r r e s p o n d i n g ~ A u t h o r: ~ C a t h e r i n e ~ D a v i s, ~ D e p a r t m e n t ~ o f ~ A n e s t h e s i o l o g y ~ a n d ~}$ Pain Medicine, Nationwide Children's Hospital, 700 Children's Drive, Columbus, OH 43205, USA. Email: cy140916@ohio.edu

doi: https://doi.org/10.14740/jmc3494 heart disease (CHD) $[2,3]$. The severity of its clinical manifestations and phenotypic expression varies based on the magnitude of the chromosomal deletion [3-6].

With an incidence of 1:15,000 - 50,000 live births, CdCS is one of the more common human chromosomal disorders [3]. Given the multi-system involvement of the disorder, anesthetic care may be required during various surgical procedures. We present a 15-month-old child with CdCS who required anesthetic care during direct laryngoscopy and supraglottoplasty. The perioperative concerns of such patients are discussed, and previous reports of anesthetic care reviewed.

\section{Case Report}

Preparation of this case report followed the guidelines of the Institutional Review Board (IRB) of Nationwide Children's Hospital (Columbus, Ohio). The patient was a 15-month-old male toddler with CdCS presenting for direct laryngoscopy and supraglottoplasty. The patient was a former 27-week, preterm infant with worsening stridor after a history of bronchiolitis 1 month ago. A previous sleep study was significant for an apnea-hypopnea index of 17 and an oxygen saturation nadir of $76 \%$. The patient was receiving home oxygen at night via a nasal cannula at $0.1 \mathrm{~L} / \mathrm{min}$. A prior echocardiogram revealed mild pulmonary stenosis. There were no recent acute illnesses or changes in physical status other than a mild increased work of breathing. Previous surgery included circumcision and left eye frontalis sling at 12 months of age to treat congenital ptosis. At that time, airway management including bag-valve-mask ventilation and endotracheal intubation were uneventful. The patient's trachea was intubated with a $3.5-\mathrm{mm}$ cuffed endotracheal tube (ETT) with a Miller 1 blade and a Cormack-Lehane grade 1 view. Preoperative home medications included budesonide $(0.5 \mathrm{mg} / 2 \mathrm{~mL})$ aerosol twice a day and as needed albuterol/ipratropium for wheezing. Vital signs were normal for age. The craniofacial structures were dysmorphic with abnormal facial features and micrognathia. Cardiovascular examination revealed a $2-3 / 6$ systolic murmur. The breath sounds were coarse with transmitted upper airway noise. The patient was held nil per os for $6 \mathrm{~h}$ and transferred to the operating room where routine American Society of Anesthesiologists' monitors were placed. Anesthesia was induced by the inhalation of incremental concentrations of sevoflurane in 50\% nitrous oxide/oxygen and a peripheral intravenous cannula was placed. The larynx was sprayed with $1 \%$ lidocaine. Direct laryngoscopy and bronchoscopy were performed during deep inhalational anesthesia with sevoflurane while main- 
taining spontaneous ventilation. Anesthesia was supplemented with incremental doses of intravenous dexmedetomidine ( 3 $\mu \mathrm{g}$, a total of five doses administered at 10 - 15 min intervals). Following diagnostic airway examination, a supraglottoplasty was performed while continuing sevoflurane anesthesia via insufflation and spontaneous ventilation. The surgical procedure lasted approximately 45 - $60 \mathrm{~min}$. Blood loss was minimal and total fluids included $100 \mathrm{~mL}$ of lactated Ringer's solution. The patient was admitted to the pediatric intensive care unit following the procedure. There was slight worsening of the stridor following the surgical procedure, but this improved overnight. Four doses of dexamethasone were administered postoperatively. On postoperative day 1, the patient was discharged to the inpatient ward. The mild inspiratory stridor and subcostal retractions continued to improve. There were some feeding concerns with a question of aspiration which required a feeding evaluation with occupational therapy and an intervention with thickening of the feedings. The remainder of the postoperative course was uneventful. The patient was discharged home on postoperative day 6 with improved air exchange and decreased stridor.

\section{Discussion}

CdCS commonly involves multiple body systems, including the upper airway (micrognathia, laryngeal and epiglottic abnormalities), cardiovascular (CHD), and central nervous system (CNS) (hypotonia) [2, 3]. Morbidity and mortality are greatest during the first year of life, with $75 \%$ of deaths occurring in the first month and about $90 \%$ occurring within the first year due to pneumonia, aspiration, and congenital heart defects [3]. After one year of age, patients have a high survival rate and life expectancy, with some individuals living into their 50 's and one into their 70's [3, 7]. Reports show that $47-75 \%$ of patients with $\mathrm{CdCS}$ will undergo a surgical procedure or diagnostic testing/imaging requiring immobilization, sedation or general anesthesia [2]. The majority of these procedures are performed in the first few years of life [5].

Anesthetic care begins with a thorough preoperative evaluation and identification of end-organ and comorbid involvement. Of particular importance during anesthesia care is the potential for difficulties with airway management due to micrognathia and upper airway abnormalities. As evident in our patient, the characteristic cry is related to the anatomical abnormalities of the larynx. The larynx, epiglottis, and glottis structures are frequently malformed. The larynx is commonly hypoplastic, narrow, and diamond-shaped [8]. The epiglottis may be small, floppy, and/or curved [8]. These anatomical abnormalities of the upper airway combined with micrognathia may lead to problems with airway management and endotracheal intubation as noted by previous reports [2, 9-12]. Although problems with airway management were not encountered with our patient, previous reports describe difficulties involving bagvalve-mask ventilation, upper airway obstruction during the induction of anesthesia, subglottic narrowing with the need to use a smaller than age-appropriate endotracheal tube, limited visualization of the glottic structures requiring video laryngoscope, laryngeal mask airway placement, or tracheostomy [2, 10-12].
Given these concerns, the appropriate equipment for dealing with the difficult airway including an indirect videolaryngoscope should be readily available prior to anesthetic induction or airway management $[13,14]$. If there are concerns regarding airway management, general anesthesia can be induced by the incremental inhalation of sevoflurane with the maintenance of spontaneous ventilation until the airway is secured or adequate bag-valve-mask ventilation is demonstrated.

Abnormal laryngeal and epiglottic anatomy in combination with neuromuscular hypotonia, particularly of the pharyngeal muscles, may lead to intermittent, partial soft tissue airway obstruction during sleep or the administration of sedative, opioid or general anesthetic agents. As demonstrated by polysomnography, our patient was diagnosed with obstructive sleep apnea (OSA). Previous reports have described postoperative respiratory events following anesthetic care in patients with CdCS [2, 12, 15]. Anatomical issues impacting upper airway control and patency may be magnified by the residual effects of anesthetic agents or the ongoing use of opioid analgesics. Furthermore, hypotonia is a frequent comorbid finding in CdCS and may further impact upper airway control $[5,16]$. Given these concerns, the use of short acting anesthetic agents should be considered. The risk of perioperative respiratory failure may be increased by pre-existing respiratory dysfunction from hypotonia, poor cough effort, chronic aspiration or recurrent pneumonia. Given the potential for postoperative events related to airway patency and respiratory function, continuous postoperative monitoring of respiratory function is suggested.

Although not required for the procedure performed in our patient, the associated hypotonia may also impact the choice of neuromuscular blocking agents (NMBAs). Patients with preexisting motor weakness and hypotonia may be sensitive to the effects of non-depolarizing NMBAs and the depolarizing agent, succinylcholine, may be contraindicated. A prolonged effect can be expected even with intermediate-acting non-depolarizing agents (atracurium, rocuronium or vecuronium) [17]. The novel reversal agent, sugammadex, offers the potential to reverse even profound neuromuscular blockade in patients with neuromyopathic conditions [18]. The limited literature available has not demonstrated increased sensitivity to the effects of NMBAs in patients with CdCS, but this possible adverse effect should be considered due to the associated hypotonia in these patients.

Despite the potential lethal side effect of hyperkalemia in susceptible populations and its association with malignant hyperthermia $(\mathrm{MH})$ and $\mathrm{MH}$-like symptoms in patients with neuromuscular disorders, succinylcholine has been administered without adverse effects to patients with $\mathrm{CdCS}[12,15$, $19,20]$. Given the limited and anecdotal experience of these reports, no definitive conclusions can be drawn regarding the safety of succinylcholine in this patient population. Additionally, a single case report suggests the possible occurrence of $\mathrm{MH}$ in a patient with CdCS [21]. The clinical symptoms occurred immediately after the induction of anesthesia with a volatile agent during attempted placement of a laryngeal mask airway. Mask ventilation had become difficult, the teeth were clenched, and the mouth could not be opened. No change was noted after the administration of propofol and succinylcholine. Associated tachycardia and an increase in body temperature from 35.7 to $37.7{ }^{\circ} \mathrm{C}$ were noted. The anesthetic was abort- 
ed, the clinical signs and symptoms resolved without the administration of dantrolene, and the remainder of his hospital course was uneventful. Many other reports have demonstrated the successful and uneventful use of volatile anesthetic agents thereby limiting the suggestion that there is any concern of $\mathrm{MH}$-susceptibility in this patient population.

A significant percentage (15-20\%) of patients with CdCS have CHD (most commonly patent ductus arteriosus, ventricular septal defect, atrial septal defect, and, tetralogy of Fallot) [5]. Although spontaneous deletions result in the majority of CdCS cases, a greater incidence and higher complexity of cardiac anomalies are noted in those with unbalanced translocations $[3,4]$. Given the high association of CHD in CdCS, a preoperative echocardiogram is suggested in all patients.

Other health problems associated with CdCS include swallowing and feeding difficulties, poor weight gain and physical growth retardation, recurrent infections such as otitis and pneumonia, orthopedic deformities including scoliosis, and involvement of the CNS (hyperacusis and abnormalities involving the brainstem and cerebellar hypoplasia) [5, 22]. Seizures have been reported in up to $10 \%$ of patients [22-24]. Preoperative management includes ensuring therapeutic anticonvulsant levels prior to the surgical procedure. Routine anticonvulsant medications should be administered the morning of the procedure despite concerns of the patient's nil per os status with subsequent intraoperative dosing as needed [25].

Joint and soft tissue contractures may lead to problems with intraoperative positioning [10, 15, 24]. Growth retardation, nutritional issues, and CNS disabilities may predispose to temperature instability with hypothermia. Thus, care must be taken to reduce the risk of intraoperative hypothermia with continuous intraoperative temperature monitoring and the use of overhead heating lights, forced airway warming, increased room temperature, and heated and humidified inspired gases.

Given the multisystem involvement of CdCS and the likelihood of the need for surgical procedures or diagnostic imaging, anesthetic care is frequently required in this patient population. The preoperative evaluation should seek to identify the associated end-organ involvement. Of particular importance are upper airway and laryngeal abnormalities with the potential for problematic airway management or difficult endotracheal intubation, associated CHD, and CNS involvement including intellectual disability, seizures, and hypotonia.

\section{Acknowledgments}

None to declare.

\section{Financial Disclosure}

None to declare.

\section{Conflict of Interest}

None to declare.

\section{Informed Consent}

In accordance with the IRB guidelines of Nationwide Children's Hospital, IRB review and written informed consent are not required.

\section{Author Contributions}

Catherine Davis prepared the initial, subsequent, and final drafts; Jonathan Grischkan did the review of final draft, perioperative care of patient; Joseph Tobias did the concept, review of all drafts, postoperative care of patient.

\section{Data Availability}

Any inquiries regarding supporting data availability of this study should be directed to the corresponding author.

\section{References}

1. Lejeune J, Lafourcade J, Berger R, Vialatte J, Boeswillwald M, Seringe P, Turpin R. [3 cases of partial deletion of the short arm of a 5 chromosome]. C R Hebd Seances Acad Sci. 1963;257:3098-3102.

2. Guala A, Spunton M, Mainardi PC, Emmig U, Acucella G, Danesino C. Anesthesia in Cri du chat syndrome: information on 51 Italian patients. Am J Med Genet A. 2015;167A(5):1168-1170.

3. Rodriguez-Caballero A, Torres-Lagares D, RodriguezPerez A, Serrera-Figallo MA, Hernandez-Guisado JM, Machuca-Portillo G. Cri du chat syndrome: a critical review. Med Oral Patol Oral Cir Bucal. 2010;15(3):e473478.

4. Overhauser J, Huang X, Gersh M, Wilson W, McMahon J, Bengtsson U, Rojas K, et al. Molecular and phenotypic mapping of the short arm of chromosome 5: sublocalization of the critical region for the cri-du-chat syndrome. Hum Mol Genet. 1994;3(2):247-252.

5. Liverani ME, Spano A, Danesino C, et al. Children and adults affected by Cri du Chat syndrome: Care's recommendations. Pediatr Rep. 2019;11:1-5.

6. Corcuera-Flores JR, Casttellanos-Cosano L, Torres-Lagares D, Serrera-Figallo MA, Rodriguez-Caballero A, Machuca-Portillo G. A systematic review of the oral and craniofacial manifestations of cri du chat syndrome. Clin Anat. 2016;29(5):555-560.

7. Guala A, Spunton M, Kalantari S, Kennerknecht I, Danesino C. Neoplasia in Cri du Chat Syndrome from Italian and German Databases. Case Rep Genet. 2017;2017:5181624.

8. Ward PH, Engel E, Nance WE. The larynx in the cri du chat (cat cry) syndrome. Laryngoscope. 1968;78(10):17161733.

9. So E, Kim S. Difficult intubation caused by an immature upper airway in a patient with cri-du-chat syndrome: a 
case report. J Dent Anesth Pain Med. 2020;20(1):49-53.

10. Carmer K. Anesthetic considerations for patient with cri du chat syndrome. Int Student J Nurse Anesth. 2015;14:43-46.

11. Castresana MR, Stefansson S, Cancel AR, Hague KJ. Use of the laryngeal mask airway during thoracotomy in a pediatric patient with cri-du-chat syndrome. Anesth Analg. 1994;78(4):817.

12. Yamashita M, Tanioka F, Taniguchi K, Matsuki A, Oyama T. Anesthetic considerations in cri du chat syndrome: a report of three cases. Anesthesiology. 1985;63(2):201202.

13. Engelhardt T, Weiss M. A child with a difficult airway: what do I do next? Curr Opin Anaesthesiol. 2012;25(3):326-332.

14. Bryant J, Krishna SG, Tobias JD. The difficult airway in pediatrics. Advan Anesth. 2013;31:31-60.

15. Brislin RP, Stayer SA, Schwartz RE. Anaesthetic considerations for the patient with cri du chat syndrome. Paediatr Anaesth. 1995;5(2):139-141.

16. Niebuhr E. The Cri du Chat syndrome: epidemiology, cytogenetics, and clinical features. Hum Genet. 1978;44(3):227-275.

17. Debaene B, Plaud B, Dilly MP, Donati F. Residual paralysis in the PACU after a single intubating dose of nondepolarizing muscle relaxant with an intermediate duration of action. Anesthesiology. 2003;98(5):1042-1048.
18. Tobias JD. Current evidence for the use of sugammadex in children. Paediatr Anaesth. 2017;27(2):118-125.

19. Martyn JA, Richtsfeld M. Succinylcholine-induced hyperkalemia in acquired pathologic states: etiologic factors and molecular mechanisms. Anesthesiology. 2006;104(1):158-169.

20. Ragoonanan V, Russell W. Anaesthesia for children with neuromuscular disease. Cont Educ Anaesth Crit Care Pain. 2010;10:143-147.

21. Amata AO. Difficult airway management and suspected malignant hyperthermia in a Child with Cri Du Chat Syndrome. Saudi J Anaesth. 2019;13(1):81-83.

22. Honjo RS, Mello CB, Pimenta LSE, Nunes-Vaca EC, Benedetto LM, Khoury RBF, Befi-Lopes DM, et al. Cri du Chat syndrome: Characteristics of 73 Brazilian patients. J Intellect Disabil Res. 2018;62(6):467-473.

23. Colover J, Lucas M, Comley JA, Roe AM. Neurological abnormalities in the 'cri-du-chat' syndrome. J Neurol Neurosurg Psychiatry. 1972;35(5):711-719.

24. dos Santos KM, de Rezende DC, Borges ZD. Anesthetic management of a patient with Cri Du Chat syndrome. Case report. Rev Bras Anestesiol. 2010;60(6):630-633, 350-631.

25. Jones CT, Raman VT, DeVries S, Cole JW, Kelleher KJ, Tobias JD. Optimizing anticonvulsant administration for children before anesthesia: a quality improvement project. Pediatr Neurol. 2014;51(5):632-640. 